\title{
Performance Analysis of Image Restoration Techniques for Dermoscopy Images
}

\author{
Mehmet Ali Altuncu \\ Department of \\ Computer Science \\ Kocaeli University
}

\author{
Fidan Kaya Gülağız \\ Department of \\ Computer Science \\ Kocaeli University
}

\author{
Fatma Selin Hangişi \\ Department of \\ Computer Science \\ Kocaeli University
}

\author{
Suhap Şahin \\ Department of \\ Computer Science \\ Kocaeli University
}

\begin{abstract}
Image enhancement and image restoration methods are widely used in most of the recent image processing studies. The main purpose of image enhancement is to remove noise from the image albeit different kinds. Therefore, in every field where image processing methods are used, image enhancement methods are more or less needed. And dermatological images, in which mostly image quality is inadequate most of the time, are one of the primary fields where these techniques are needed. Dermatologists that work in this field carry out the recording of wound images via a digital dermoscopy device. Later they realize the decision-making process using the image processing techniques through software. And in this study, the comparison of different image preprocessing methods is carried out in order to remove the effects of lighting and to make the software used give more accurate decisions.
\end{abstract}

\section{Keywords}

Image Restoration, Dermoscopy Images, Image Quality Assessment

\section{INTRODUCTION}

Dermatologic disorders are seen in almost all people epidemically nowadays. There are many different paths followed by dermatologists in diagnosis and treatment of dermatologic disorders. In the phase of diagnosis, deciding only through visual inspection results in success at the rate of $60 \%$, especially in the skin cancer [1]. Therefore, in order to have the diagnosis more accurate, a method called dermoscopy came forward. Dermoscopy is a dermatological examination method used to diagnose the dark-colored spots. Processing the image by means of software used with the devices used for this purpose and having a more accurate diagnosis is aimed. The operation of dermoscopy is a topic that attracts many different researchers' attention for many years.

In 2002, a study was carried by Piccolo et. al. [2] in order to investigate the effectiveness of the computer-based diagnosis method. According to the study, it is seen that the error rate of the computer-based diagnosis is $26 \%$ where this rate is $0.6 \%$ for an experienced doctor. In 2005, an Internet-based dermatologic disease diagnosis system was generated for the first time by Ivatomi et. al. [3]. With the system generated, dermatologic diseases could be determined with high success rates. In 2007, diagnosis of skin lesions was tried to be carried out via cellular phones [4]. In the end of the study, it was shown that diagnosis done through cellular devices gave out more accurate results depending on the enhancement applied on the images. In 2010, a device that is capable of recording dermoscopy images through mobile devices is developed for the first time. This device was named, Handoscopy [5]. By means of this system, it was provided that people who are not experts could also get dermatological images and could transfer these images to the remote diagnosis systems. In 2011, a study, that shows how accurate results will be given by the usage of the dermoscopy systems developed for dermatologists in developing countries like Africa.It was shown that despite the problems of Internet connection usage of computers in the developing countries, success rates up to seventy percent can be obtained [6]. In the forthcoming years, whether the dermatological images got by smart phones are enough or not for the diagnosis was investigated by Asaid et. al. With the study, it was shown that images got by clinical photography are more of high quality but smart phones also get images that contain adequate information for the diagnosis [7].After proving that mobile devices can be used in this field, studies aimed at enhancing the images obtained with these devices were carried out. In 2014, with the study, which aimed to decrease the blurriness in the images, carried out by João et. al. [8], the effect of image enhancement on the results gathered was shown in the clear. In 2016, the problems of blurriness and irregular lighting in dermatological images were mentioned by Xie et. al. [9]. A new method is suggested to prevent the image distortion occurred because of these reasons.

With the studies carried out, it is seen that technology is also effective in the field of dermatology. Mobile devices, also being used in the diagnosis of dermatological diseases, the quality of the image obtained has become a really important determinant. However, the medium that the image was taken or blurriness and lighting problems that can occur for different reasons would have critical effects in the process of diagnosis. When studies carried out for this purpose are examined, it is seen that the studies are mostly on detection of hair in the image, removing the factor of hair or on the operation of classification [9]. With this study, unlike the other studies carried out in the previous year, different image enhancement techniques are used to decrease the effect of the shadow in the dermoscopic images and the success of the methods are tested on the dermatological images.

The remainder of this paper is organized as follows. Section 2 presents the detailed explanation of image restoration methods that used to improve dermoscopy images. Section 3 explains the dataset and evaluation metrics Section 4 presents experimental and comparative results of the image restoration techniques. Section 5 is the last section, draws a conclusion and suggests some future works.

\section{BRIEF OVERVIEW}

By using filters in image processing, operations as deblurring on the image, bringing some details into open, softening the 
image, edge sharpening or edge finding can be realized. Filters, generally, applied as hovering a core matrix over the entire image and retrieving the product of the multiplications for each pixel. The filtering operation is used as a preprocessing step and it makes the future operations to be conducted more comfortable.

There are linear and non-linear kinds of filters used in image processing. Linear filters are rather appropriate for some specific noise models and these types of filters are more prone to blur the image. The most widespread used examples to the linear filters are the Average filter and Gaussian filter. And non-linear filters are mostly used in the areas where the linear filters are inadequate. Applications of non-linear filters are harder than linear filters and are generally used for some (specific) purposes like edge concretizing. The most distinct methods of non-linear filtering can be given as mean filter, median filter, and wiener filter.

Within the context of the study, the effect of the shadow, which is formed as a result of different reasons in the dermatological images obtained using mobile devices, is tried to be decreased by applying both linear and non-linear filters. For this purpose, non-linear median filter, adaptive median filter and adaptive weighted median filter and linear Gaussian blur filter are used. The detailed explanation of the methods is given in the proceeded chapters.

\subsection{Median Filter}

The most widespread method used for decreasing the noise in the salt-pepper noise images is the median filter. Median filter, which is nonlinear, gives out successful results in the mediums of speech processing, image processing, and impulsive noise, where linear filters fall short [10]. The median filter acts like the low-pass filter to block all of the high-frequency elements like the noise in the image and thus, the image gets blurred [11]. In the median filter, the pixel values of the image are hovered over a sliding window. The picture's pixel values in the size that constitute the window are sorted in an ascending order and the middle value is assigned to the central pixel [12].

$$
\begin{aligned}
& \begin{array}{|l|l|l|}
\hline 65 & 140 & 255 \\
\hline 43 & 184 & 57 \\
\hline
\end{array} \\
& \begin{array}{|l|l|l|l|l|l|l|l}
443 & 184 & 57 & 35435765 & 110 & 140 & 184210255 \longrightarrow & \longrightarrow
\end{array} \\
& \begin{array}{|l|l|l|}
\hline 35 & 210 & 110 \\
\hline
\end{array}
\end{aligned}
$$

Fig 1: Median filter method

\subsection{Adaptive Median Filter}

The adaptive median filter is one of the methods that effectively decreases the spotted noises on the medical ultrasonic images. The most important advantage of this method can be thought to be protecting the image information while decreasing the noise. Differently from the other median filter methods, in the adaptive median filter, the size of the window changes during the filtering operation [13, 14]. Adaptive median filter algorithm works in two steps.

\section{Step 1:}

$$
\begin{aligned}
& M_{x_{1}}=M_{\text {med }}-M_{\text {min }} \\
& M_{x_{2}}=M_{\text {max }}-M_{\text {med }}
\end{aligned}
$$

If $M_{x_{1}}>0$ and $M_{x_{2}}<0$ process passes to second step.
Else, the window size is increased and step 1 is repeated

Step 2:

$$
\begin{aligned}
& M_{y_{1}}=M_{\text {gray }}-M_{\text {min }} \\
& M_{y_{2}}=M_{\text {max }}-M_{\text {gray }} \\
& \text { If } M_{y_{1}}>0 \text { and } M_{y_{2}}<0, M_{\text {gray }} \text { is designated as output. }
\end{aligned}
$$

Else $M_{\text {med }}$ is designated as output.

In formula $1, M_{\min }$ value represents the minimum gray value of the pixels in the filtering window, $M_{\max }$ value represents the maximum gray value of the pixels in the filtering window. In formula $2, M_{\text {med }}$ value represents the median gray value of the pixels in the filtering window and $M_{\text {gray }}$ value represents the gray value of the center pixel in the filtering window.

\subsection{Adaptive Weighted Median Filter}

Adaptive weighted median filter algorithm is used in the cases where the standard median filter algorithm is inadequate. Also in the adaptive weighted median filter algorithm, the size of the window that encloses each pixel is variable, different than the standard median filter algorithm [16]. The algorithm suggested in the study consists of two phases like the algorithm [17]. In the first phase, whether there is a pixel that is exposed to noise on the image is determined. If there is noise on the pixel, it passes to the second phase and the pixel is changed with the weighted median value. If not, the pixel value remains the same with the image pixel value. The flow chart that belongs to the adaptive weighted median filter is shown in Fig 2.

\subsection{Gaussian Blur Filter}

2D Gaussian Blur method is a method that is used commonly to decrease the noise and details on the image. But this method is also sued as a pre-processing phase in the artificial vision algorithms with the intent of strengthening the structures of the images in different magnitudes [18]. Gaussian Blur can be defined as the convulsion of the core matrix sampled from the Gaussian function by the image pixels. Convulsion in discrete time can be defined as below [19].

$$
\begin{aligned}
& y(n)=x(n)^{*} h(n)=\sum_{k=-\infty}^{\infty} x(k) h(n-k) \\
& \varphi(x)=\frac{1}{\sigma \sqrt{2 \pi}} \mathrm{e}^{-\frac{x^{2}}{2 \sigma^{2}}}
\end{aligned}
$$

Here, $x$ represents the input image, $y$ the output image and $h$ the core matrix. In the operation of convulsion, the window magnitude of the pixels in the input image and the core matrix is the same. If the image output value calculated as a result of the operation is not an integer, it is rounded off to an integer. As a result of using different core matrixes in the convulsion operation, different filtering operations are carried out on the image. And Gauss function can be defined as: 


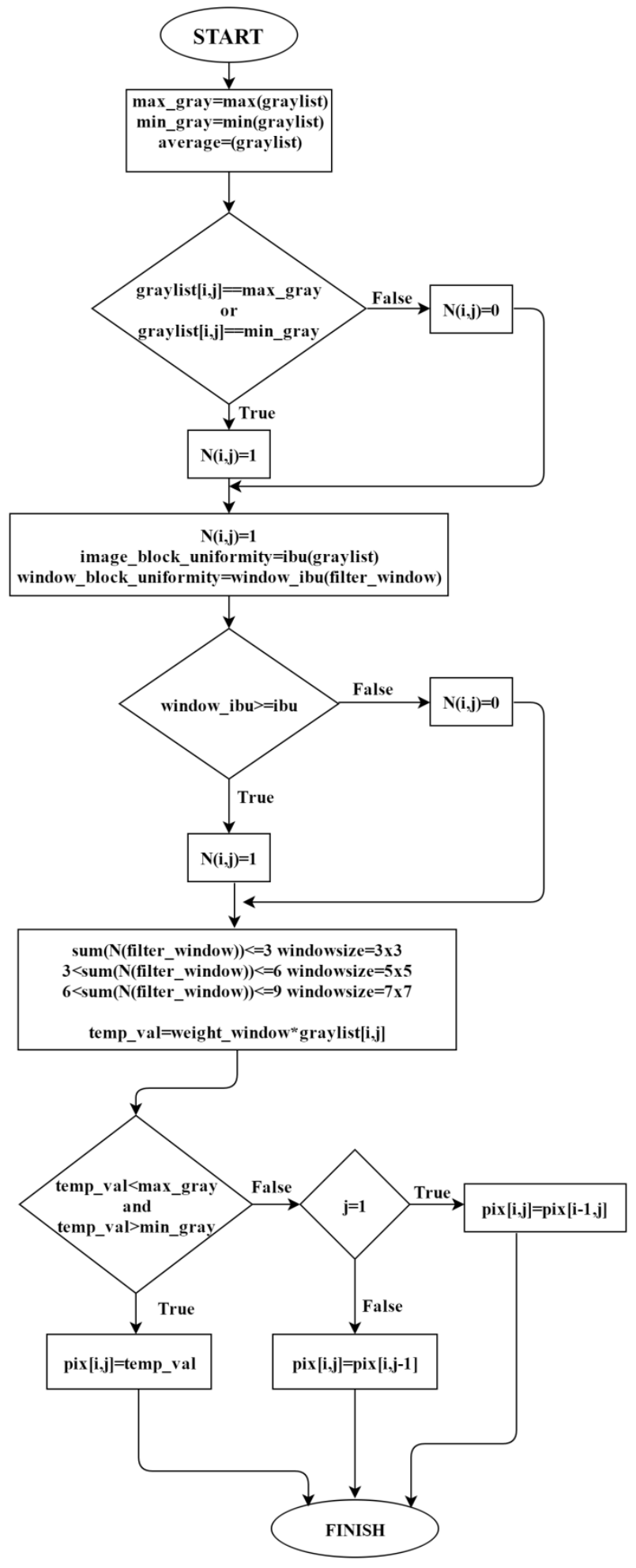

Fig 2: Adaptive Weighted Median Filter algorithm

Here is stated to be the standard deviation of the distribution [20]. In order to the Gauss structure of the filter to be preserved value must increase in parallel with the core matrix magnitude. In other words, Gauss core coefficients are interdependent with the value. The example core matrixes of Gauss filter are shown in Fig 3.

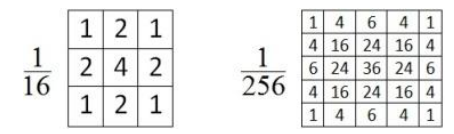

Fig 3: $3 \times 3$ and $5 \times 5$ dimensional Gaussian kernel matrices

\section{DATASET AND EVALUATION TECHNIQUES}

In this study, the effect of the shadow formed on the dermatological images obtained by the mobile devices is tried to be decreased. But in the literature, there is no database generated with the intent of enhancing the quality of the dermatological images [8]. The studies in the literature are generally conducted by adding different noises on the images in the databases that have unimpaired images. In our study, unlike existing studies, enhancement is tried to be carried out on twenty-six different images obtained by the patients using mobile devices. For this purpose, Samsung S6 and LG G3, mobile phones which have 12 megapixels of resolution maximum. Images in different resolutions are obtained via these phones. Previously, it was shown that dermatological images obtained using mobile devices, branded Samsung [6] and iPhone [7] having 5 megapixels of resolution, could be used in the diagnosis of the diseases. But as the mobile devices used in these studies are not compatible with the current technology, within the context of the study, an up-todate dataset is created. In Fig 4, some example images that belong to the dataset created are shared.
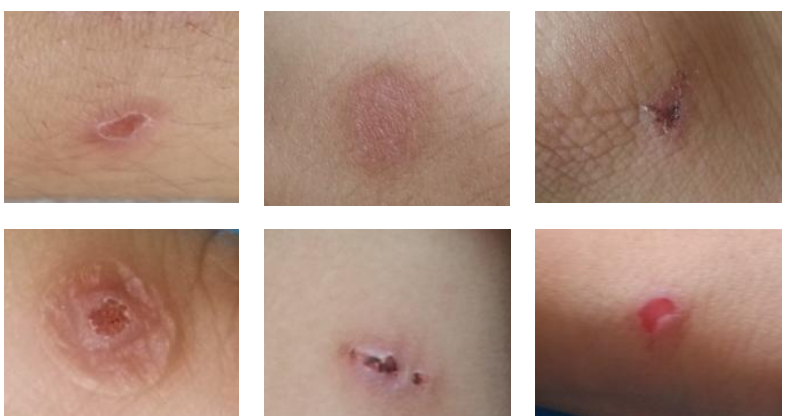

Fig 4: Sample images that belongs to created dermoscopy images dataset

While evaluating the success of the filters applied to decrease the effect of the shadow in the wound images taking place in the dataset obtained three different methods are used. These are Mean Square Error (MSE), Peak Signal-to-Noise Ratio and Correlation Index (CI). The formula for MSE and PSNR are given by the following equations (5), (6) and (7).

$$
M S E=\frac{1}{M N} \sum_{n=1}^{M} \sum_{m=1}^{N}\left[g^{\prime}(n, m)-g(n, m)\right]^{2}
$$

The MSE method is the most widespread method used to evaluate the images on which the restoration operation is applied. $g^{\prime}(n, m)$ value taking place in the Formula 5 represents the image obtained after the restoration operation and the $g(n, m)$ value the original image. The MSE method can give different error results depending on the resolution of the image.

$P S N R=-10 \log _{10} \frac{M S E}{S^{2}}$ 
The $\mathrm{S}$ value taking place in the Formula 6 represents the biggest pixel taking place in the image. The unit of measure of the PSNR value is $\mathrm{dB}$. The PSNR value is an ideal method to restoration operations applied on the same image to be evaluated.

$$
r=\frac{\sum_{i}\left(x_{i}-x_{m}\right)\left(y_{i}-y_{m}\right)}{\sqrt{\sum_{i}\left(x_{i}-x_{m}\right)\left(y_{i}-y_{m}\right)}}
$$

The correlation index is one of the alternative methods that are used to evaluate the operations carried out on the image. The value $x_{i}$ shown in Formula 7 represents the intensity of the $i$. pixel of the first one from the images whose correlation index will be calculated, $y_{i}$ value, the intensity of the $i$. pixel of the second one from the images whose correlation index will be calculated, $x_{m}$ value, the mean value of the intensity of the first image, and the $y_{m}$ value, the mean value of the intensity of the second image. In the next chapter MSE, PSNR and CI values obtained using the original image and the restored image is given.

\section{EXPERIMENTAL RESULTS}

Filters that will be used within the context of the study to decrease the effect of the shadow are developed using Phyton language. Application of the filters and obtaining results are also done using Phyton environment. In Table 1 and Table 2, results belonging to the images in the dataset having the highest and lowest resolutions are given.

Table 1. Performance comparison results for most corrupted image

\begin{tabular}{|l|c|c|c|c|}
\hline Metrics & Median Filter & Adaptive Median Filter & Adaptive Weighted Median Filter & Gaussian Blur Filter \\
\hline MSE & 37.022152 & 39.752286 & 55.419178 & 44.930384 \\
\hline PSNR & $\mathbf{3 2 . 4 4 6 1 8 6}$ & 32.137182 & 30.694202 & 31.605402 \\
\hline CI & 0.993975 & 0.993258 & 0.993269 & 0.993953 \\
\hline Time Elapsed & $7.238999 \mathrm{sec}$. & $125.549000 \mathrm{sec}$. & $64.375999 \mathrm{sec}$. & $6.744999 \mathrm{sec}$. \\
\hline
\end{tabular}

Table 2. Performance comparison results for least corrupted image

\begin{tabular}{|l|c|c|c|c|}
\hline Metrics & Median Filter & Adaptive Median Filter & Adaptive Weighted Median Filter & Gaussian Blur Filter \\
\hline MSE & 6281.422030 & 6244.557930 & 6145.461117 & 6227.850857 \\
\hline PSNR & 10.150223 & 10.175786 & $\mathbf{1 0 . 2 4 5 2 5 8}$ & 10.187421 \\
\hline CI & 0.668387 & 0.666483 & 0.659708 & 0.668412 \\
\hline Time Elapsed & $13.970000 \mathrm{sec}$. & $237.462000 \mathrm{sec}$. & $123.293999 \mathrm{sec}$. & $13.476000 \mathrm{sec}$. \\
\hline
\end{tabular}

As it is seen in the tables, the performances of the filtering methods vary depending on the resolution of the image. In the images with low resolution, Median Filter, Adaptive Median Filter has a lower error rate and higher PSNR values compared to Adaptive Weighted Filter and Gaussian Blur Filters. And in the images with how resolution Adaptive Weighted Median Filter has the best performance. In Fig 5, from the images in the dataset, the image in which the shadow's effect is the highest and the resolution is the lowest (Image 1) and the image in which the shadow's effect is the lowest and the resolution is highest (Image 2) are presented. The first row in Fig 5 shows the original versions of the images. And the rows below demonstrate the images obtained after applying the filters. When the images are compared, it is seen that Adapted Weighted Median Filter method decreases the effect of the shadow for the images with low resolution. But for the images with high resolution, it is seen that there is no need to apply the filters. And when the filters applied are compared in terms of their processing times it is observed that Gaussian Blur and Median Filter are the ones that carry out the operation within the shortest time. As the methods of Adaptive Median Filter and Adaptive Weighted Median Filter require additional operations, even though they decrease the rate error, require longer processing time.

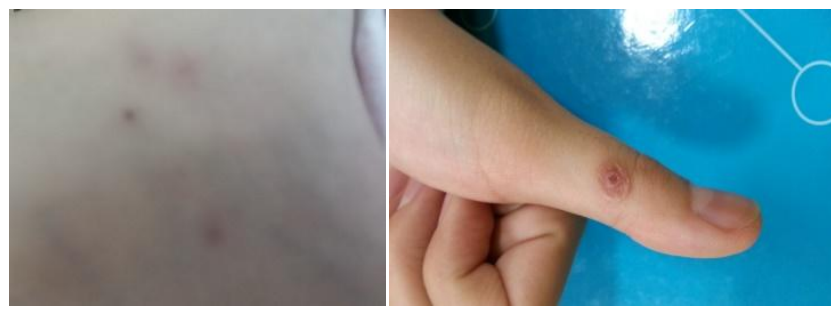

Original Image 1

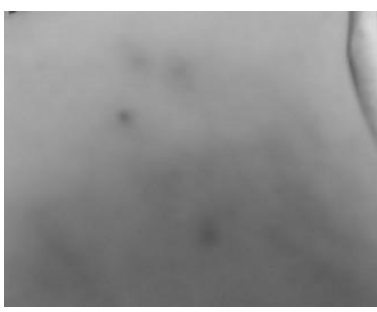

Median Filter

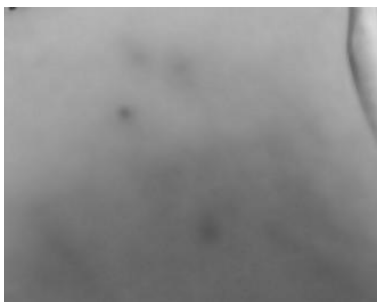

Adaptive Median Filter

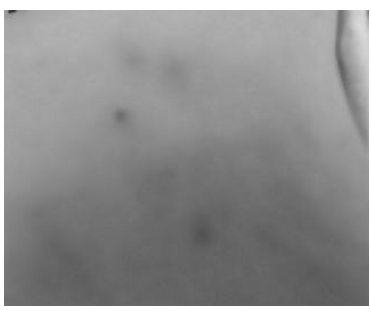

Adaptive Weighted Median Filter
Original Image 2

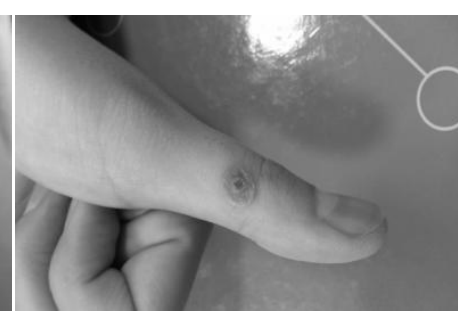

Median Filter

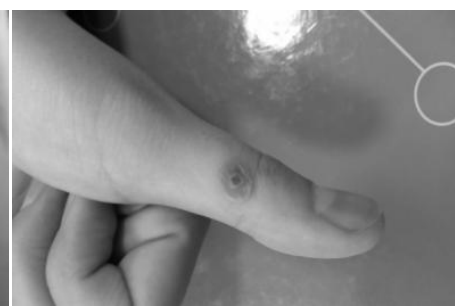

Adaptive Median Filter

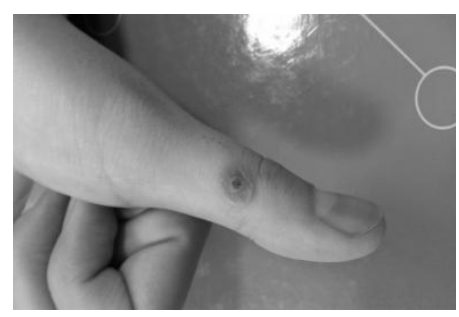

Adaptive Weighted Median Filter 


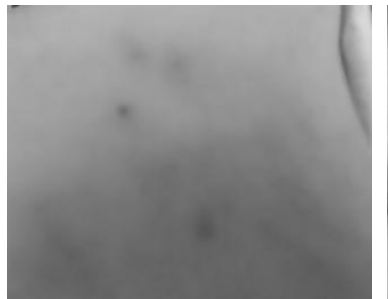

Gaussian Blur Filter

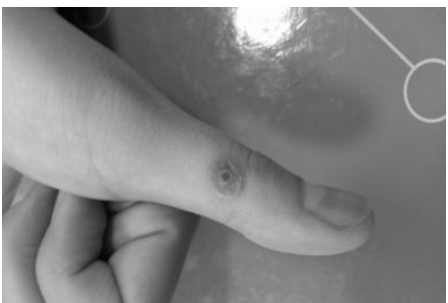

Gaussian Blur Filter
Fig 5. Simulation results for different images

\section{CONCLUSION}

Enhancement operations in the medical images with insufficient image quality are important and challenging operation. There are many different methods that can be applied on the images for this purpose.

Within the context of the study, the effect of the shadows on the image are tried to be decreased by applying different filters to enhance the images with different resolutions. It is seen that four filters that are applied for this purpose have variant effects on images with different resolutions. Application of filters on the images with a high resolution where distortions very low did not have a serious benefit but it was observed that the best method for decreasing the shadow on the images with low resolution is the Adaptive Weighted Median Filter.

\section{REFERENCES}

[1] H. Kittler, H. Pehamberger, K. Wolff, "Diagnostic accuracy of dermoscopy", The Lancet Oncology,vol. 3, no. 3, pp. 159-165, 2002.

[2] D. Piccolo, A. Ferrari, K. Peris, R. Daidone, B. Ruggeri, S. Chimenti, "Dermoscopic diagnosis by a trained clinician vs. a clinician with minimal dermoscopy training vs. computer-aided diagnosis of 341 pigmented skin lesions: a comparative study", British Journal of Diagnosis, vol. 147, no. 3, pp. 481-486, 2002.

[3] Iyatomi, H., Oka, H., Hashimoto, M. 2005. An internet based melanoma diagnostic system - toward the practical application. In Proceedings of theIEEE Symposium onComputational Intelligence in Bioinformatics and Computational Biology.

[4] C. Massone, R. H. Wellenhof, V. A. Siess, G. Gabler, C. Ebner, H. P. Soyer, "Melanoma screening with cellular phones", PLoS ONE, vol. 2, no. 5, pp. 1-4, 2007.

[5] Handyscope-mobile dermatoscope: Handyscope (2010). [Online]. Available:http://www.handyscope.net

[6] K. Tran, M. Ayad, J. Weinberg, A. Cherng, M. Chowdhury, S. Monir, M. El Hariri, C. Kovarik, "Mobile teledermatology in the developing world: Implications of a feasibility study on 30 Egyptian patients with common skn diseases", Journal of the American Academy of Dermatology, vol. 64, no. 2, pp. 302-309, 2011.

[7] R. Asaid, G. Boyce, G. Padmasekara, "Use of a smartphone for monitoring dermatological lesions compared to clinical photography", Journal of Mobile Technology in Medicine, vol. 1, no. 1, pp. 16-18, 2012.

[8] M. João, M. Vasconcelos, L. Rosado, "No-reference blur assessment of dermatological images acquired via mobile devices", Image and Signal Processing, vol. 8509, pp. 350-357, 2014.

[9] F. Xie, Y. Lu, A. C. Bovik, Z. Jiang, R. Meng, "Application-driven no-reference quality assessment for dermoscopy images with multiple distortions", IEEE Transaction on Biomedical Engineering, vol. 63, no. 6, pp. 1248-1256, 2016.

[10] Lei, Wang, Yuan Yibao, and Piao Weiying. "A Two-Step Robust Filter for Mean Line Extraction Based on the Median Filter" 2015 Fifth International Conference on Instrumentation and Measurement, Computer, Communication and Control (IMCCC), 2015.

[11] Sunil, K., Kasturiwale,H. 2016. Quality assessment of Median filtering techniques for impulse noise removal from digital images. In Proceedings of theInternational Conference on Advanced Computing and Communication Systems .

[12] Zhao, J., Botao, Z., Hongjun, L. 2011. A median filter FPGA with harvard architecture. In Proceedings of the International Conference on Information Science and Technology.

[13] A. Yang, "Research on the ultrasonic medical image filtering method to combine mathematical morphology with adaptive median filter", Electronic Measurement Technology, vol. 8, 2009.

[14] Li, L., Meng, X., Liang, X. 2013. Reduction of impulse noise in MRI images using block-based adaptive median filter.In Proceedings of theIEEE International Conference on Medical Imaging Physics and Engineering.

[15] Chenguang, Y., Liu, Y. 2010. Application of modified adaptive median filter for impulse noise. In Proceedings of theInternational Conference onIntelligent Control and Information Processing.

[16] Wang, C. Y., Li, L., Yang, F. P., Gong, F. 2010. A new kind of adaptive weighted median filter algorithm. 2010.In Proceedings of theInternational Conference on Computer Application and System Modeling .

[17] P.Geetha, B. Chitradevi, "Image denoising using adaptive weighted median filter in synthetic aperture radar images", International Journal of Computer Science and Information Technology Research, vol. 2, no. 3, pp. 413-420, 2014.

[18] Shapiro, L. G.,Stockman, G., "Computer Vision" 1st Edition: Prentice Hall, 2001.

[19] Gedraite, E. S., Murielle, H. 2011. "Investigation on the effect of a Gaussian Blur in image filtering and segmentation", In Proceedings of theELMAR.

[20] B. Marhaba, Z.Mourad, K. Wassim, "Image restoration using a combination of blind and non - blind deconvolution techniques", International Journal of Engineering Research \& Science, vol. 2, no. 5, pp. 225239, 2016. 